\title{
Veränderungen der Sommerwitterung im südlichen Mitteleuropa von 1270-1400 als Auftakt zum Gletscherhochstand der Neuzeit
}

\section{Zusammenspiel von anthropogenen und natürlichen Datentypen in der Witterungs- und Klimageschichte des Mittelalters}

In den dreieinhalb Jahrhunderten vor der Schaffung staatlicher Meßnetze kann sich die Klimageschichte in der Schweiz fast vollständig auf anthropogene Datentypen abstützen. Frühe Meßreihen, serielle Daten aus Witterungstagebüchern und summarische Beschreibung des Witterungscharakters überlappen, kontrollieren und ergänzen sich zu einer zeitlich und räumlich recht hohen Dichte der Aussage. Zusammen mit biologischen und physikalischen Daten erlauben sie es, die Temperatur- und Niederschlagsverhältnisse in den einzelnen Kalendermonaten grob abzuschätzen. Unter den natürlichen Daten lassen sich einzig die Dichtewerte des Spätholzsegments in Baumringen als Indikator für die Spätsommertemperaturen sinnvoll integrieren (PFISTER, 1984a, 1984b).

Für die Zeit des Hoch- und Spätmittelalters sind weit weniger einschlägige Quellen vorhanden, die zudem in der Regel nur sporadisch Informationsfragmente enthalten. Unter diesen Voraussetzungen sind bestenfalls noch saisonale Aussagen sinnvoll und möglich. Größere Beobachtungslücken müssen immer wieder durch Quellen aus weiter entfernten Räumen überbrückt werden, was die Gefahr von Fehlschlüssen birgt. Der Rückgriff auf die in Form säkularer Serien vorliegenden Messungen der Spätholzdichte ist in dieser Übergangsphase der ausklingenden anthropogenen Information in doppelter Weise hilfreich: Einmal ist anzunehmen, daß sich anthropogene Daten und Dichtewerte aus derselben Klimaprovinz ergänzen, stützen und kontrollieren. Dann gewährleisten die radio-dendrochronologischen Daten ein Minimum an Information für Jahre mit anthropogenen Aufzeichnungslücken, oder sie liefern Anhaltspunkte, um den Analogiewert von Beobachtungen aus benachbarten Klimaräumen zu überprüfen.

\section{Der Aussagewert der radio-dendrochronologischen Daten für die Rekonstruktion der Sommerwitterung}

Die Eignung von radio-dendrochronologischen Daten als Indikatoren der Sommerwitterung ist von FLOHN (1985) am Beispiel der Serie von Lauenen (sCHWEINGRUBER et.al., 1978, 1979) untersucht worden, die auch für die vorliegende Arbeit herangezogen wird; die Lauenen-Reihe zeigt zwar gute Übereinstimmungen mit jener aus dem österreichischen Ötztal (LAMPRECHT, 1978), die Korrelation mit den für die AprilSeptember-Temperatur besonders repräsentativen $(r=0.83)$ Serie von Weinlesedaten aus Mittel- und Westeuropa (LE ROY LADURIE, BAULAND, 1980) liegt jedoch unter 0.7, und die Kalibration mit der Basler Temperatur-Reihe (BIDER, SCHÜEPP, V.RUDLOFF, 1958) ergab nur für den Spätsommer befriedigende Resultate.

Da im Mittelalter nur größere Ausschläge der Sommerwitterung einigermaßen vollständig erfaßt werden können, ist die Übereinstimmung der Lauenen-Reihe mit solchen Ereignissen im vierhundertfünfzigjährigen Zeitraum 1525-1979 anhand der Temperatur- und Niederschlagsindices für den Sommer überprüft worden (PFISTER, 1984a). Zu diesem Zwecke sind die Dichtewerte in sieben Stufen gegliedert worden:

\begin{tabular}{rl}
\multicolumn{1}{l}{ Dichte } & Index \\
\hline$<880$ & -3 \\
$880-919$ & -2 \\
$920-959$ & -1 \\
$960-1039$ & 0 \\
$1040-1079$ & 1 \\
$1080-1119$ & 2 \\
$>=1120$ & 3
\end{tabular}

Die Berechnungen wurden für kühle und warme Sommer getrennt durchgeführt (Tab. la und 1b).

Aus den Gegenüberstellungen (Tab. 1a, 1b) bestätigt sich erneut, daß zwischen Dichtewerten und Sommertemperaturen signifikante Zusammenhänge bestehen, wobei - wie aus dem für rechteckige Tafeln geeigneten Assoziationsmaß Kendall's Tau b (LOETHER, Mc TAVISH, 1974: 229, 256) hervorgeht - kühle Sommer von den Dichtewerten offenbar etwas zuverlässiger erfaßt werden als warme Sommer. Wie weit die Abweichungen im Einzelfall gehen können, zeigt das

Christian Pfister, PD Dr.,

Historisches Institut der Universität Bern, Engehaldenstr. 4, 3012 Bern 
Tabelle 1a Spätholzdichte und Temperaturindices in kühlen Sommern (Gewi-T-Index <0) 1525-1979

(ab 1755 beruhen die Indices auf Temperaturmessungen)

Spätholzdichte

\begin{tabular}{|l|l|l|l|l|}
\hline $\begin{array}{l}\text { Gewichtete } \\
\text { T-Indices Sommer }\end{array}$ & \multicolumn{1}{|c|}{-3} & \multicolumn{1}{|c|}{-2} & \multicolumn{1}{|c|}{-1} \\
\hline$<--6$ & $\begin{array}{l}|c| \\
1579,1621,1628,1816, \\
1860,1956\end{array}$ & $\begin{array}{l}1542,1585,1675,1805, \\
1838\end{array}$ & $1813,1821,1882$ & $\begin{array}{l}1588,1639,1812,1815, \\
1841,1888,1913\end{array}$ \\
\hline-5 bis -3 & $\begin{array}{l}1576,1587,1594,1597, \\
1627,1698,1829,1833\end{array}$ & $\begin{array}{l}1527,1529,1573,1593, \\
1692,1814,1880,1954, \\
1978\end{array}$ & $\mathrm{~N}=12$ & $\mathrm{~N}=49$ \\
\hline-1 bis -2 & $1851(1969)$ & $\begin{array}{l}1614,1640,1714,1740, \\
1835(1975),(1977)\end{array}$ & $\mathrm{N}=33$ & $\mathrm{~N}=224$ \\
\hline
\end{tabular}

Jahre in Klammer: eine Depression der Werte durch Umweltbelastung wird vermutet (SCHWEINGRUBER et. al, 1983)

Kendall's TAU B 0.32

Signifikanz $<0.0000$

Tabelle 1b Spätholzdichte und Temperaturindices in warmen Sommern (Gewi-T-Index > 0) 1525-1979 (ab 1755 beruhen die Indices auf Temperaturmessungen)

Spätholzdichte

\begin{tabular}{|l|l|l|l|l|}
\hline $\begin{array}{l}\text { Gewichtete } \\
\text { T-Indices Sommer }\end{array}$ & \multicolumn{1}{|c|}{3} & \multicolumn{1}{|c|}{2} & \multicolumn{1}{c|}{0} \\
\hline$=6$ & - & 1536,1540 & $\begin{array}{l}1556,1718,1826,1834, \\
1846\end{array}$ & $\begin{array}{l}1534,1616,1719,1859, \\
1904,1947,1950,1952\end{array}$ \\
\hline 5 bis 3 & - & $1666,1774,1781,1921$ & $\mathrm{~N}=19$ & $\mathrm{~N}=43$ \\
\hline 1 bis 2 & - & $\begin{array}{l}1681,1708,1766,1777, \\
1779,1791,1800,1901, \\
1917,1919,1931\end{array}$ & $\mathrm{~N}=54$ & $\mathrm{~N}=201$ \\
& & & \\
\hline
\end{tabular}

Kendall's TAU B 0.12 Signifikanz $<0.009$

Beispiel der Jahrhundertsommer 1616, 1719 und 1947, die überhaupt nicht durch erhöhte Spätholzdichten hervortreten (vgl. Tab. 1b) und jenes der sehr kalten Sommer 1812, 1813 und 1841, für welche die Temperatur durch Messungen zweifelsfrei ermittelt worden ist und wo offenbar eine annähernd normale Spätholzbildung möglich war. Im weitern zeichnen sich Jahre mit extrem tiefen Frühjahrstemperaturen (1614, 1714, $1740,1835,1975)$ und normaler Sommerwitterung (PFISTER, 1984b) durch tiefe, solche mit stark überdurchschnittlichen Temperaturen im Mai-Juni und anschließend durchwegs unterdurchschnittlichen Temperaturen (1931) durch hohe Dichtewerte aus. Der Vergleich mit den Niederschlagsindices ergab für die trockenen Sommer schwach signifikante Werte (Kendalls Tau b -0.09), nicht dagegen für die nassen Sommer.

Aus diesem Überblick läßt sich der Schluß ziehen, daß Messungen der Spätholzdichte in alten Hölzern mit einiger Vorsicht als Indikator für deutliche Ausschläge der Sommertemperaturen herangezogen werden können. Die Ergebnisse sollten aber wenn immer möglich durch den Vergleich mit anthropogenen Daten erhärtet werden. Für die sommerlichen Niederschlagsverhältnisse, denen sowohl für die "glaziale» wie für die agrarische Konjunktur ebenfalls erhebliche Bedeutung zukommt, bleibt dagegen vorläufig die Aussage der Schriftquellen allein maßgebend.

\section{Die mittelalterlichen Schriftquellen}

Die in alten Chroniken und Annalen enthaltenen Witterungsaufzeichnungen sind seit jeher klimageschichtlich ausgewertet worden. Vom letzten Jahrhundert an haben Naturwissenschafter wie ARAGO (1858), HENN!G (1904), AMBERG $(1890,1892,1892)$ und zuletzt DARNAJOUX (1976), um nur die einschlägigsten zu 
nennen, umfangreiche Kompilationen zusammengetragen, die teilweise quantitativ umgesetzt worden sind (EASTON, 1928, FAIR BRIDGE, 1967). Erst seit einem knappen Jahrzehnt ist Alarm geschlagen worden, hat es sich doch gezeigt, daß ein hoher Prozentsatz der in diesen Kompilationen enthaltenen Aufzeichnungen falsch datiert, inhaltlich entstellt, stillschweigend aus weit entfernten Klimaprovinzen übernommen oder schlicht und einfach erfunden worden ist (ALEXANDRE, 1976; BELL, OGILVIE, 1978; INGRAM et.al., 1981; PFISTER 1984a; ALEXANDRE, 1986). Wie gering der Wert gängiger Kompilationen für das Mittelalter einzustufen ist, hat die Überprüfung des Werks von AMBERG $(1890,1892,1897)$ gezeigt, wo nach ALEXANDRE (1986) der Anteil der fehlerhaften Meldungen $50 \%$ erreicht, aber $30 \%$ der vorhandenen, zuverlässigen Berichte nicht erfaßt worden sind.

Was die sorgfältige Messung und Kalibration bei der Prüfung quantitativer Daten, das ist die Quellenkritik beim Umgang mit deskriptiver Information. Die Textkritik richtet sich auf die Urheberschaft des vorgegebenen Verfassers und auf die Entstehungszeit, die inhaltliche Kritik auf das Verhältnis zwischen dem Wortlaut der Quelle und den geschilderten Tatsachen. Wer diesen Forderungen für die Periode des Mittelalters gerecht werden will, nimmt eine Sisyphusarbeit auf sich. Einmal enthalten zahlreiche Werke Beschreibungen der Witterung und ihre Auswirkungen auf die belebte und unbelebte Umwelt, aber meist nur sporadisch, etwa in Form eines Hinweises auf einen besonders süßen oder sauren Jahrgang, so daß der Aufwand zur Gewinnung einiger Körner von hochkarätiger Information mit jenem eines Goldwäschers vergleichbar ist. Dann ist häufig die Urheberschaft anonym oder knifflig zu ermitteln; manchmal waren mehrere Schreiber beteiligt; schließlich schöpften die Chronisten mit besonderer Vorliebe aus anderen Quellen, um ihre Erzählung möglichst eindrücklich auszuschmücken, so daß ein und derselbe verregnete Sommer oft in Dutzenden von Quellen in mehr oder weniger starker Verzerrung auftaucht. Der belgische Forscher Pierre ALEXANDRE (1986) hat sich der entbehrungsreichen Aufgabe unterzogen, für die Periode bis 1425 sämtliche einschlägigen gedruckten Quellen aus West- und Mitteleuropa (ohne England, aber inklusive das Gebiet der heutigen CSSR, Schlesiens und Norditaliens) zu sichten, kritisch zu prüfen, die klimageschichtliche Information daraus auszuziehen und zu einer Kompilation zusammenzustellen, der ersten, die für die Periode des Mittelalters ausschließlich auf zuverlässiger Information beruht. ALEXANDRE berücksichtigt nur Originaltexte von Augenzeugen oder Zeitgenossen der Ereignisse sowie Aufzeichnungen, die aus (Original-)Quellen stammen, welche heute verloren sind. Als besonders fündig erwiesen sich städtische Chroniken, Annalen von Klöstern, seltener Tatenberichte (Gesta) oder Lebensbeschreibungen (Vitae) von Bischöfen oder Äbten sowie Beschreibungen von Wundern (Miracula).
Für den Vergleich mit der schweizerischen Gletschergeschichte sind primär die Daten aus Schwaben und der (Deutsch-)Schweiz, jene aus der Westschweiz und Burgund sowie jene aus Bayern und Franken herangezogen worden (vgl. Karte in ALEXANDRE, 1986); in einzelnen Jahren mußte auf Beobachtungen aus entfernteren Räumen (Holland, Lothringen, Rheinland) zurückgegriffen werden.

Die räumliche Dichte ist sehr ungleich:

Unter dem Begriff "Sources très attentives au climat» hat ALEXANDRE (1986) jene Quellen zusammengefaßt, die mindestens vier meteorologische "Ereignisse von längerer Dauer» (Monate, Jahreszeiten) in verschiedenen Jahren in maximal zweijährigen Abständen beschreiben. Dazu gehören für unseren Raum unter anderem die "Annales Colmarienses» (1278-1297, 1301-1305), zwei Konstanzer Chroniken (1348-1353, 1370-1382), die "Annales Basilienses» (1267-1277) sowie die Augsburger Chronik (1370-1388). Allerdings haben selbst diese städtischen Chroniken über meteorologische Langzeitphänomene zu wenig dicht und kontinuierlich Buch geführt, als daß Beobachtungslücken im Sinne "normaler Verhältnisse» interpretiert werden dürften.

Insgesamt haben sich für den Raum Schwaben-(Deutsch-)Schweiz 70 Quellen mit über 300 Beobachtungen, für die Westschweiz und Burgund nur gerade 8 Quellen mit 16 Beobachtungen gefunden. Das in der Periode 1525-1700 auffallende OstWest-Gefälle (PFISTER, 1984a: 23) reicht ganz offensichtlich bis ins Mittelalter zurück. Bayern-Tirol und Franken-Hessen nehmen diesbezüglich eine Mittelstellung ein.

\section{Die Rekonstruktion der Sommerwitterung durch die Synthese von natïrlichen und anthropogenen Daten}

In Tabelle 2 sind die in Dichtestufen umgesetzten radio-dendroklimatischen Daten von Lauenen (SCHWEINGRUBER, 1978) zusammen mit den einschlägigen Witterungsaufzeichnungen (ALEXANDRE, 1986) dargestellt. Sie werden ergänzt durch Erläuterungen der savoyardischen "Landvögte» im Unterwallis zum Ausfall der Wein- und Getreidezehnten, welche uns freundlicherweise durch Dr.Pierre Dubuis, Sion, übermittelt worden sind. Die Abrechnungen erfolgten in natura; auffallende Mindererträge waren dem Zehntherrn gegenüber zu begründen, desgleichen Aufwendungen für die Reparatur von Mühlen, die von Überschwemmungen beschädigt, oder von Dächern, die vom Sturm weggetragen worden waren. Im vorliegenden Kontext beschränkt sich die Auswertung auf Bemerkungen, die Schlüsse auf die sommerlichen Witterungsverhältnisse erlauben, beispielsweise Dürreschäden bei Reben (1327) und Getreide (1352, 1361). Für Jahre mit positiven oder negativen Abweichungen der Spätholzdichte von $>=2$ oder solchen mit Beschreibungen, die auf einen extremen Witte- 
Tabelle 2 Die Beurteilung der Temperatur- und Niederschlagsverhältnisse in außergewöhnlichen Sommern auf Grund von Daten aus Schriftquellen und dendro-klimatischen Daten 1270-1400

\begin{tabular}{|c|c|c|c|c|c|}
\hline Jahr & Daten aus Schriftquellen & Quellen & Spätholzdichte & $\begin{array}{l}\text { Indice } \\
\mathrm{T}\end{array}$ & ies $\mathrm{N}$ \\
\hline 1270 & trocken (Bayern), 13.7. reife Trauben (Basel), 1.8. Überschw. Rhein & $\mathrm{B} 14, \mathrm{~S} 51$ & +3 & +2 & -2 \\
\hline 1273 & warmer Herbst (Basel) & S51 & +3 & +2 & \\
\hline 1274 & Regen bis 1.8., dann schön und warm, Weinlese verspätet (Basel) & S51 & 0 & -1 & +1 \\
\hline 1275 & Überschwemmung Rhein Anfang Juli (Basel) & & -2 & -1 & +1 \\
\hline 1276 & $\begin{array}{l}\text { Rebenblüte Ende Mai, Erntebeginn 8.7., Blüte von Bäumen im Aug. } \\
\text { (Basel) }\end{array}$ & S51 & -1 & +1 & \\
\hline 1278 & gute Weinqualität, trocken (Konstanz) & S11 & +1 & +1 & -2 \\
\hline 1280 & naß (Rouen), Überschwemmung (Bayern), Regen (Colmar) & N2, S51 & +1 & -1 & +2 \\
\hline 1287 & Dürre den ganzen Sommer (Champagne), Weinlese früh (Colmar) & $\mathrm{CH} 8, \mathrm{~S} 51$ & +3 & +3 & -3 \\
\hline 1290 & kalt und naß (Colmar) & S51 & -1 & -2 & +2 \\
\hline 1291 & gute Weinqualität (Colmar, Konstanz) & S11, S51 & +2 & +1 & \\
\hline 1293 & warm und trocken, reichliche und qualitativ gute Weinernte (Colmar) & S51 & +1 & +2 & -2 \\
\hline 1294 & - & & +2 & +1 & \\
\hline 1296 & - & & +2 & +1 & \\
\hline
\end{tabular}

\begin{tabular}{|c|c|c|c|c|c|}
\hline Jahr & Daten aus Schriftquellen & Quellen & Spätholzdichte & $\begin{array}{l}\text { Indice } \\
\mathrm{T}\end{array}$ & $\mathrm{es}$ \\
\hline 1300 & - & & +3 & +2 & \\
\hline 1301 & - & & +2 & +1 & \\
\hline 1302 & $\begin{array}{l}\text { kalt, Überschwemmung des Rheins im Aug., wenig und saurer Wein } \\
\text { (Mainz, Colmar), Weinlese verspätet (Aquitanien) }\end{array}$ & $\mathrm{R} 29, \mathrm{~S} 51, \mathrm{Aq} 6$ & -3 & -3 & +3 \\
\hline 1303 & $\begin{array}{l}\text { Vegetation stark verfrüht, reichliche und qualitativ gute Weinernte } \\
\text { (Colmar), sehr trockener Sommer (Appenzell) }\end{array}$ & S51, Wetter & 0 & +1 & -2 \\
\hline 1304 & $\begin{array}{l}\text { sehr trocken und warm (Mainz, Colmar), Vegetation stark verfrüht, } \\
\text { reichliche und qualitativ gute Weinernte }\end{array}$ & $\mathrm{R} 29, \mathrm{~S} 51$ & +2 & +2 & -2 \\
\hline 1306 & - & & -2 & -1 & \\
\hline 1312 & - & & +2 & +1 & \\
\hline 1314 & - & & -2 & -1 & \\
\hline 1315 & $\begin{array}{l}\text { sehr kalt und fast ununterbrochen Regen (Flandern, Wallonien, lle de } \\
\text { France), extrem späte Weinlese, wenig und qualitativ schlechter Wein } \\
\text { (Bretagne), Ernteausfall in den Bergen wegen übermäBiger Nässe } \\
\text { (Saxon VS) }\end{array}$ & \multicolumn{2}{|c|}{$\begin{array}{l}\text { W23, Pc18, Pc28, - } 3 \\
\text { F116, Br5, Dubuis }\end{array}$} & -3 & +3 \\
\hline 1316 & Überschwemmungen Ende Juni, Anfang Juli (Bayern, Salzburg) & B32, B36 & +1 & & +1 \\
\hline 1317 & - & & +2 & +1 & \\
\hline 1319 & - & & +3 & +2 & \\
\hline
\end{tabular}

\begin{tabular}{|c|c|c|c|c|}
\hline Jahr & Daten aus Schriftquellen & Quellen & Spätholzdichte & ${ }_{\mathrm{T}}^{\text {Indices }} \mathrm{N}$ \\
\hline 1325 & kein Regen (Picardie, Holland) & Pc22, H5 & +1 & $+1-1$ \\
\hline 1326 & Trockenheit in Normandie, Flandern, Holland, Wein billig in Konstanz & $\begin{array}{l}\text { W41, N18, H5, } \\
\text { S11, Dubuis }\end{array}$ & +1 & $+1-1$ \\
\hline 1327 & $\begin{array}{l}\text { Minderertrag der Reben in Saillon VS «propter siccitatum temporis" } \\
\text { (Trockenschäden) }\end{array}$ & & 0 & $+1-2$ \\
\hline 1330 & $\begin{array}{l}\text { sehr starke Vegetationsverspätung, schlechte Reifung des Weins } \\
\text { (Picardie), kalter Sommer (Holland), Wein schlecht und teuer (Konstanz) }\end{array}$ & $\mathrm{P} 10, \mathrm{P} 19, \mathrm{H} 5$ & +1 & $-1+1$ \\
\hline
\end{tabular}




\begin{tabular}{|c|c|c|c|c|c|}
\hline Jahr & Daten aus Schriftquellen & Quellen & Spätholzdichte & $\begin{array}{l}\text { Indice } \\
T\end{array}$ & ${ }^{\mathrm{ces}} \mathrm{N}$ \\
\hline 1331 & $\begin{array}{l}\text { Mitte März bis Anfang Juni kein Regen (Paris), } 12 \text { Wochen ohne Regen, } \\
\text { gute Weinqualität (Zürich) }\end{array}$ & Pc30, S37 & +3 & +3 & -3 \\
\hline 1333 & $\begin{array}{l}\text { reichliche Weinernte, gute Qualität (Wallonien, Picardie, Paris), } \\
\text { große Hitze während der Weinlese (Paris) }\end{array}$ & $\begin{array}{l}\text { W42, Pc19, } \\
\text { Pc30 }\end{array}$ & +3 & +2 & -1 \\
\hline 1335 & $\begin{array}{l}\text { geringe Weinernte, schlechte Reifung, nasser August (Wallonien, } \\
\text { Picardie, Franken), Minderertrag der Reben in Chillon VS wegen } \\
\text { Mißwachs in diesem Jahr }\end{array}$ & $\begin{array}{l}\text { W42, Pc19, F13 } \\
\text { Dubuis }\end{array}$ & -3 & -3 & +2 \\
\hline 1336 & Dürre (Wallonien, Paris), sehr gute Weinqualität (Zürich) & W42, Pc30, S9 & +3 & +3 & -2 \\
\hline 1337 & - & & -2 & -1 & \\
\hline 1338 & $\begin{array}{l}\text { Einfall von Wanderheuschrecken in Mitteleuropa (Konstanz), geringe } \\
\text { Weinernte (Schaffhausen) }\end{array}$ & S10, S9 & -2 & -1 & \\
\hline 1342 & $\begin{array}{l}\text { naß (Bayern, Lindau), schwere Überschwemmungen in ganz West- und } \\
\text { Mitteleuropa }\end{array}$ & B3, S9 & +1 & -1 & +3 \\
\hline 1343 & $\begin{array}{l}\text { sehr große Niederschläge: Juni, Ende Juli, Ende Aug. und Anfang Sept. } \\
\text { (Lindau), Überschwemmung Bodensee, Limmat, Rhein, zahlreiche Erd- } \\
\text { rutsche (Appenzell) }\end{array}$ & $\begin{array}{l}\text { S9, S10, S37, } \\
\text { S48, S66, Wetter }\end{array}$ & -1 & -1 & +3 \\
\hline 1345 & $\begin{array}{l}\text { sehr kalt und naß, sehr schlechter Wein (Picardie), Überschwemmungen } \\
\text { (Bayern) }\end{array}$ & Pc30, F1 & -3 & -3 & +3 \\
\hline 1346 & $\begin{array}{l}\text { sehr kalt und naß April bis Juni, nach dem 2. Aug. warm, Rebenblüte am } \\
\text { 2. Aug. noch nicht beendet (Lindau) }\end{array}$ & S9 & -3 & -3 & +1 \\
\hline 1347 & $\begin{array}{l}\text { sehr naß, Reben haben anfangs September (!) noch nicht verblüht, } \\
\text { 8-10 Tage mit Schneedecke anfangs Oktober (Lindau) }\end{array}$ & s9 & -2 & -3 & +3 \\
\hline 1348 & warm (Franken, Konstanz) & $\mathrm{F} 13, \mathrm{~S} 10$ & 0 & +1 & \\
\hline 1350 & $\begin{array}{l}\text { ziemlich warmer und schöner Sommer, nasser August (Wallonien), } \\
\text { Regen im August (Poitou), gutes Wetter das Jahr über (Konstanz) }\end{array}$ & W42, F9, S10 & -3 & -1 & +1 \\
\hline 1352 & $\begin{array}{l}\text { sehr warm und trocken (Wallonien), Minderertrag an Getreide in } \\
\text { Riddes VS «propter siccitatum temporis» (Trockenschäden) }\end{array}$ & W42, Dubuis & -1 & +1 & -2 \\
\hline 1353 & warmer und trockener Juni (Konstanz), reichliche Weinernte (Strassburg) & S10, S65 & +1 & +1 & -1 \\
\hline 1355 & - & & +2 & +1 & \\
\hline 1356 & kalt, nur August etwas wärmer (Mainz), schlechter Wein (Mainz, Köln) & R9, R31 & -1 & -2 & \\
\hline
\end{tabular}

\begin{tabular}{|c|c|c|c|c|c|}
\hline Jahr & Daten aus Schriftquellen & Quellen & Spätholzdichte & $\begin{array}{l}\text { Indice } \\
T\end{array}$ & $\mathrm{es}_{\mathrm{N}}$ \\
\hline 1357 & $\begin{array}{l}\text { große Hitze während eines Teils des Jahres, Hitze } \\
\text { (wohl Trocken-)Schäden in den Reben (Konstanz) }\end{array}$ & S10 & -1 & +1 & -2 \\
\hline 1359 & Juli bis anfangs September regnerisch (Picardie, Mainz) & Pc22, R31 & -3 & -3 & +3 \\
\hline 1361 & $\begin{array}{l}\text { trocken und heiß, Weinlesebeginn 1. Sept. (Konstanz), Trockenschäden } \\
\text { am Getreide in Riddes VS }\end{array}$ & S10 Dubuis & 0 & +2 & -2 \\
\hline 1364 & $\begin{array}{l}\text { häufige Gewitter, im Juli } 10 \text { Tage sehr kalt (Mainz), April, Mai schön, Juni, } \\
\text { Juli kalt, August warm (Strassburg) }\end{array}$ & $\mathrm{R} 31, \mathrm{~S} 68$ & +1 & $-1-$ & +1 \\
\hline 1366 & Regen und kalt von April bis Ende August, Überschwemmungen (Mainz) & R31 & -3 & -3 & +3 \\
\hline 1369 & reichliche Weinernte, gute Qualität (Mainz) & R31 & +1 & +1 & \\
\hline 1370 & $\begin{array}{l}\text { Mai dürr, dann regnerisch und kalt, wenig und schlechter Wein (Mainz), } \\
\text { guter Wein (Konstanz, Basel) }\end{array}$ & R31, S12, S48 & -3 & -3 & +3 \\
\hline 1371 & heiß und sehr trocken (Mainz), Wein von guter Qualität (Mainz, Konstanz) & $\mathrm{R} 31, \mathrm{~S} 12$ & 0 & +2 & -2 \\
\hline 1374 & $\begin{array}{l}\text { Regen Mitte Juni bis August, geringe Weinernte (Mainz), lange Regen- } \\
\text { periode während eines Teils des Jahres (Basel, Straßburg), August } \\
\text { schön (Basel) }\end{array}$ & R31, S48, S68 & +1 & & +2 \\
\hline 1375 & $\begin{array}{l}\text { Juni, Juli, August sehr trocken und warm (Mainz), } 14 \text { Wochen ohne } \\
\text { Regen bis im August (Basel) }\end{array}$ & $\mathrm{R} 31, \mathrm{~S} 48$ & +1 & +3 & -3 \\
\hline
\end{tabular}




\begin{tabular}{lllrr}
\hline Jahr & Daten aus Schriftquellen & Quellen & Spätholzdichte & $\begin{array}{l}\text { Indices } \\
\text { T }\end{array}$ \\
\hline 1376 & reichliche Weinernte von guter Qualität (Mainz, Konstanz) & R31, S12 & +1 & +1 \\
1377 & sehr kalt bis im Juni, schlechtes Wetter bis Oktober (Augsburg) & S5 & -1 & $-1+1$ \\
1379 & regnerisch und warm (Mainz), reichliche Weinernte (Konstanz) & R31, S12 & +1 & $+1+1$ \\
\hline 1382 & $\begin{array}{l}\text { sehr warmes Frühjahr, Vegetationsvorsprung, Juni kalt (Mainz), Sommer R31, W12 } \\
\text { kalt und naß (Wallonien) }\end{array}$ & R31, Pc23 & +2 & -1 \\
1383 & $\begin{array}{l}\text { bis 15. August trocken, dann Regen und kalt (Mainz, Picardie) } \\
1385\end{array}$ & $\begin{array}{l}\text { kein Regen von Anfang Mai bis Oktober (Besançon), frühe Weinlese } \\
\text { (Dijon, Beaune), kein Strohertrag in Liddes VS }\end{array}$ & Bg5, Bgll, Bgl & +1 \\
1387 & kalt und regnerisch (Mainz, Augsburg), schlechter Wein (Mainz) & R31, S5 & 0 & -2 \\
\hline 1391 & - & & -2 & $-2+2$ \\
1393 & sehr heißer Sommer, kein Regen während 16 Wochen (Bern, Zürich) & S37, S44 & +2 \\
\hline
\end{tabular}

Quellen:

Wetter handschriftliche Appenzeller Chronik im Besitze von Dr. H. Stricker, Bern

Dubuis “Ernteberichte» der Landvögte, der Grafen von Savoyen, mitgeteilt von Dr. P. Dubuis, Université de Lausanne

Buchstaben und Zahlen Quellenangaben aus ALEXANDRE, 1986

rungsverlauf schließen lassen, sind die Sommerperioden mit ungewichteten thermischen und hygrischen Indices beurteilt worden. Sie können für den einzelnen Monat Werte von $-1,0,+1$, für die gesamte Jahreszeit folglich +3 resp. -3 annehmen (vgl. PFISTER 1984a: 112). Die Extremwerte blieben jenen Sommern vorbehalten, für welche die Aussagen der radiodendrochronologischen und der deskriptiven Daten einigermaßen übereinstimmen. Wo einschlägige $\mathrm{Be}$ schreibungen fehlen, wurden die um 1 verminderten Dichtestufen der Spätholzdaten als Indices eingesetzt; sofern die Chronisten nicht angeben, wie lange eine Kälte-, Hitze-, Dürre- oder Regenperiode andauerte, wurden die Indices +2 resp. -2 eingesetzt. Dies gründet auf der Annahme, daß Witterungsanomalien, die der Aufzeichnung würdig scheinen, mindestens zwei Monate angedauert haben.

Die Schwächen dieser stark subjektiv gefärbten Quantifizierung liegen auf der Hand: wir verfügen weder über annähernd vollständige Daten noch über einheitliche Kriterien der Gewichtung. Beobachtungen aus Wallonien (Liège) und dem Rheinland (Mainz), auf die für manche Jahre zurückgegriffen werden mußte, dürften die Situation im südlichen Mitteleuropa nicht immer adäquat wiedergeben. Dies gilt vor allem für den Niederschlag; die Temperaturen sind über Distanzen von mehreren hundert Kilometern, auch zwischen Berg- und Talstationen, mit Koeffizienten in der Größenordnung von 0.9 hoch signifikant korreliert (MESSERLI, 1979; FLOHN, 1985). Im ganzen gesehen stimmen radio-densitometrische und anthropogene Daten erstaunlich gut überein:
1. In Jahren mit sehr geringen Dichtewerten werden weitere aus der Periode seit 1525 bekannte typische Symptome des «Eiszeitsommers» erwähnt: Vegetationsverspätung, kleine Weinernte, geringer Zuckergehalt des Weinmosts. Wie solche Sommer in den Alpen ausgesehen haben, wissen wir von gut dokumentierten Jahren wie 1628 und 1816 her: 15- bis 20malige Bildung von Neuschneedecken unter $2000 \mathrm{~m}$, mehrere Schneefälle bis ins höhere Mittelland, im Extremfall (4. Juli 1587) bis auf $450 \mathrm{~m}$ hinunter, keine Schneeschmelze oberhalb von 2000-2400 m, eine Vegetationsverspätung von einem Monat und mehr (PFISTER, 1984a: 141). Innerhalb der Untersuchungsperiode zeigen diese "Jahre ohne Sommer» eine bemerkenswert symmetrische zeitliche Verteilung: 1302, 1315, 1330?, 1335, 1345-1347, 1350?, $1359,1366,1370$. Das heißt, sie treten in den ersten 30 Jahren überhaupt nicht auf, dann setzen sie vereinzelt ein, erreichen in den 1340er-Jahren mit drei Fällen einen Höhepunkt, nach 1350 werden sie seltener, zwischen 1370 und 1400 fehlen sie wiederum vollständig. Im Mittel der Periode 1525-1979 tritt die Dichtestufe -3 alle 30 Jahre auf; eine mit den Jahren 1300-1370 vergleichbare Häufung von «Eiszeitsommern" finden wir nur gerade in den drei Dezennien 1579-1600. Von der Größe der Kälteanomalien her fallen die Jahre 1346 und 1347 aus dem Rahmen: 1346 war die Rebenblüte in Lindau nach dem 2. August noch nicht beendet. Eine Vegetationsverspätung in dieser Größenordnung ist seit dem frühen 16. Jahrhundert nur gerade in den Jahren 1628 und 1816 nachzuweisen (PFISTER, 1984b). Im folgenden Jahr 
hatten die Reben in Lindau selbst anfangs September noch nicht verblüht. Dies deutet auf die Kälteanomalie im Juli und August hin, die innerhalb der letzten fünf Jahrhunderte einzigartig ist. Einmalig innerhalb der gesamten Serie von Lauenen ist auch das Muster der Dichtestufen in den Jahren 1345-1347: -3, -3, -2; das heißt, das "Ausbleiben" des Sommers in drei unmittelbar aufeinanderfolgenden Jahren.

2. Die Dichtestufe $+3(\geqq=1120$ ) ist zwischen 1270 und 1336 in acht Jahren erreicht worden; in den 650 Jahren seither dagegen nur noch gerade ein einziges Mal; nämlich im Jahr 1473. Wir wissen nicht, inwieweit diese Daten auf ein Witterungsmuster hindeuten, das für die Zeit des Hochmittelalters typisch war, aber in der Folge nur noch vereinzelt aufgetreten ist. Die vorliegenden Witterungsberichte sind für das dazu benötigte zeitliche und elementspezifische Auflösungsvermögen zu lakonisch.

Innerhalb der Periode seit 1525 dürfte ihm am ehesten das Jahr 1540 mit seiner vom März bis zum Jahresende fast ununterbrochenen Schönwetterperiode entsprechen (PFISTER: 1984a: 138). Auch in ungünstigen Lagen konnte in diesem Jahr eine quantitativ und qualitativ hochwertige Weinernte eingebracht werden. Eine größere Häufigkeit von Sommern dieses Typs, bei einem weitgehenden Fehlen von naß-kalten Perioden, könnte sehr wohl die im Hochmittelalter wesentlich weiter nach Norden reichende Verbreitung des Weinbaus erklären (LAMB, 1977: 276-279).

Für einige Jahre zeigen sich Widersprüche zwischen radiodensitometrischen Daten und den Aufzeichnungen der Chronisten:

- Drei warme und trockene Sommer (1371, 1375, 1385) sind in den Dichtewerten nicht adäquat abgebildet, was in Anbetracht analoger Beispiele in der Eichperiode (u.a. 1616) kaum erstaunt (vgl. Tab. lb).

- Der Sommer 1382 wird trotz einer hohen Dichte als kalt beschrieben. Dies dürfte, wie 1931, auf den Vegetationsvorsprung im Frühsommer zurückzuführen sein (vgl. Tab. 1b).

- Keine Erklärung kann für den Sommer 1330 Vegetationsverspätung, wenig Wein von schlechter Qualität, aber überdurchschnittlicher Dichtewert angeboten werden. Dasselbe gilt für 1350: die densitometrischen Daten deuten auf Kälte hin, wogegen der Chronist von Konstanz gutes Wetter meldet.

\section{Die Klimaschwankung von 1342-1347 und der Vorstoß des Aletschgletschers}

Die Rekonstruktion der Gletschergeschichte aus Aufzeichnungen von Bildquellen hat für die Periode 1525-1860 im Anschluß an die Synthese der verschiedenen Datentypen sozusagen eine Kalibration der daraus abgeleiteten Indexwerte auf klimageschichtlicher Ebene erlaubt (FURRER et al., 1980; ZUMBUEHL, 1980; GAMPER, SUTER, 1982; PFISTER, 1984a, HOLZHAUSER, 1984a, 1984b).

Mit Hilfe von fossilen Bäumen, die im Gletschervorfeld in situ gefunden werden, ist uns die einmalige Möglichkeit gegeben, frühere Gletschervorstöße, von denen keine historischen Quellen berichten, sowohl zeitlich als auch in ihrem Ausmaß zu erfassen (HOLZHAUSER, 1984b). Damit sind die Voraussetzungen für eine witterungsgeschichtliche Feininterpretation gegeben. Mit Radiokarbondaten hat HOLZHAUSER (1984a) einen Hochstand des Aletschgletschers um $1350 \pm 60$ Jahre belegt. Auch am Rhonegletscher ist neuerdings im 14. Jahrhundert ein Hochstand nachgewiesen worden (freundl. Mitteilung Dr. Holzhauser und Dr. Zumbühl). Beim Aletschgletscher darf als gesichert gelten, daß der Hochstand kaum über das Jahr 1400 hinaus gedauert hat. Um 1412 nämlich beginnt im Bereich der Hochstandsmoränen eine Arve zu wachsen, die beim Vorsto $B$ im 17. Jahrhundert vom Gletscher umgedrückt und in die Moräne einsedimentiert worden ist (HOLZHAUSER, 1984a). Auf der Basis der vorliegenden Daten soll im folgenden versucht werden, diesen Vorstoß klimageschichtlich zu interpretieren und zeitlich näher einzugrenzen.

Zungenbewegungen des Großen Aletschgletschers sind weit schwieriger zu interpretieren als solche von kleineren Eisströmen. Infolge ihrer außerordentlichen Länge reagiert die Zunge nicht auf kurzfristige Veränderungen der klimatischen Bedingungen. So integriert der Hochstand von 1653 zwei, wenn nicht drei, gletschergünstige Phasen (1563-1579, 1585-1597, 1618-1629) (HOLZHAUSER, 1984a).

In Figur 1 sind die auf Tabelle 2 beruhenden Zehnjahresmittel der ungewichteten thermischen und hygrischen Indices dargestellt.

Für die Jahre vor 1320-1330 deuten weder die densitometrischen Daten von Lauenen noch die anthopogenen Aufzeichnungen auf Bedingungen hin, die einen kräftigen Vorsto $B$ der Gletscherzunge vor der Jahrhundertmitte befördert haben könnten. Die warmen und trockenen Monate dominieren, das nasse Jahrzehnt 1310-19 bleibt Episode, in den 1330er Jahren stehen drei Sommerperioden mit hohen Dichtewerten im Wechsel mit einem, möglicherweise zwei (1330?, 1335) Eiszeitsommern, was auf eine große Variabiliät hindeutet. Ausreichend für die Auslösung eines weitreichenden Vorstoßes scheinen dagegen die Bedingungen in den Jahren 1342-1347: sehr hohe Niederschlagsmengen in den Sommern 1342 und 1343 , vergleichbar vielleicht mit dem Jahr 1588 , in welchem Mitteleuropa - bei 77 Niederschlagstagen in Luzern - sozusagen ohne Unterbruch in der Bahn der atlantischen Störungen lag (DOUgLAS, LAMB, 1979; PFISTER, 1984a). Darauffolgend eine einzigartige Serie von drei naßkalten Sommern, von welchen der letzte, 1347, mit einiger Wahrscheinlichkeit kälter war als jener von 1816. Für den Massenhaushalt des Glet- 
schers dürfte die Summationswirkung dieser naßkalten Phase stark ins Gewicht gefallen sein, die Tatsache, daß die im Verlaufe von mehreren aufeinanderfolgenden Akkumulationsperioden angehäuften Schneemassen der Ablation fast völlig entzogen wurden, wie dies auch in den Jahren 1815-16 der Fall war (PFISTER, 1984a: 132). Als mäßig gletschergünstig sind die Sommerperioden 1350-1370 zu beurteilen. Nach 1380 dominieren warm-trockene Sommer.

Wenn wir die verzögerte Reaktion im Zungenbereich berücksichtigen, ist anzunehmen, daß der Gletscher im Zeitraum um 1380 einen Hochstand erreicht hat. Dies würde, wenn wir den Impuls der Jahre 1342-1347 als ausschlaggebend annehmen, im zeitlichen Verlauf weitgehend der aus der ersten Hälfte des 19. Jahrhunderts bekannten Dynamik entsprechen: der damalige Hochstand ist 1850 , etwas mehr als 30 Jahre nach der maßgebenden Klimaschwankung von 1812-1817, erreicht worden. Die klimatischen Bedingungen zwischen 1380 und 1400 begünstigen ein Rückschmelzen des Eises. Ob die Zeit bis 1412 ausgereicht hat, um die Voraussetzungen für das Wachstum der

Figur 1 Die sommerlichen Temperatur- und Niederschlagsverhältnisse im südlichen Mitteleuropa von 1270-1400 aufgrund von radio-densitometrischen Daten und anthropogenen Aufzeichungen.

Dargestellt sind die Dezennienmittel der ungewichteten Temperatur- und Niederschlagsindices

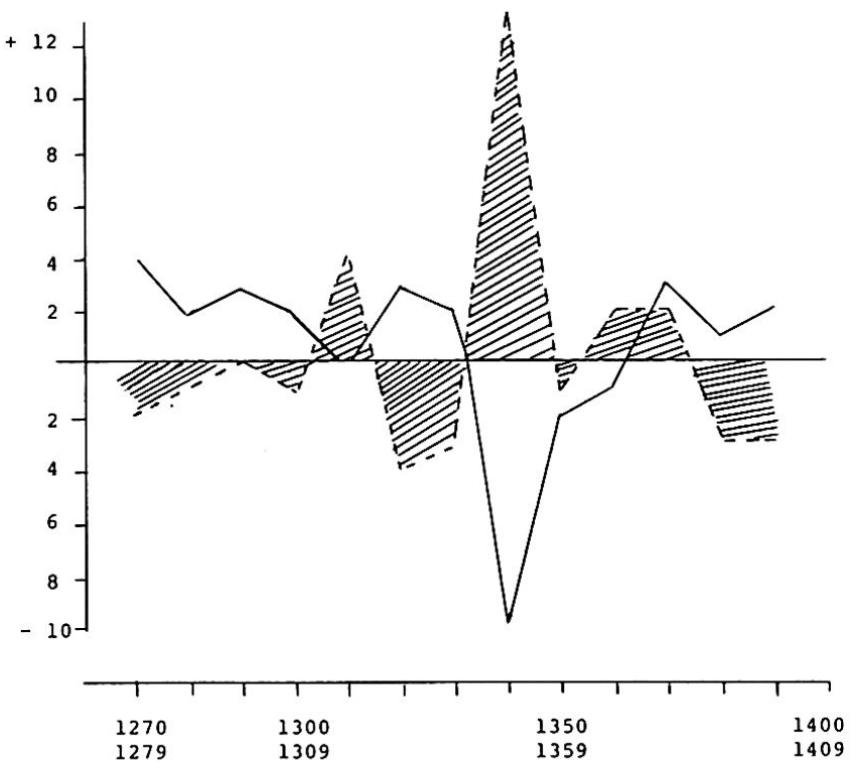

Quellen:

- radio-densitometrische Daten: SCHWEINGRUBER, 1978

- anthropogene Daten: ALEXANDRE, 1986

- Methodik der Indexbewertung: PFISTER, 1984a

- ungewichteter Temperaturindex

----- ungewichteter Niederschlagsindex besagten Arve im Zungenbereich zu sçhaffen, kann nicht entschieden werden.

Die vorliegenden Ergebnisse stehen mit der von HOLZHAUSER (in diesem Heft) auf Grund von densitometrischen Messungen am Riedgletscher postulierten Chronologie in einem gewissen Widerspruch. Die maximale Depression der Spätholzdichten liegt bei diesem Holzfund in den Jahrzehnten vor 1300, während die in der Serie von Lauenen hervortretende Baisse der 1340er Jahre nur schwach angedeutet ist. Für einen Überhang von kalten Sommern in den Jahren 1270-1300 lassen sich keine anthropogenen Belege finden. Dies kann nicht mit der Lückenhaftigkeit der Dokumentation erklärt werden, weil gerade für jene Zeit mit den "Annales Basilienses» und der "Annales Colmarienses» Quellen zur Verfügung stehen, in welchen die Beschreibung von Witterungsereignissen einen verhältnismäßig breiten Raum einnimmt (ALEXANDRE, 1986). Eine Serie von naßkalten Sommern wäre wegen der damit verknüpften Preissteigerungen und Hungersnöten von diesen Chronisten nicht übersehen worden.

Nicht auszuschließen ist dagegen, daß Veränderungen in anderen Jahreszeiten, beispielsweise feuchtere Winter- und Frühjahrsperioden, ein gewisses Vorrükken der Gletscherzunge in der ersten Jahrhunderthälfte gefördert haben: die Trends im Ausaperungszeitpunkt des Säntis-Gipfels von 1821-51 und von 1886-1980 stimmen mit den Gletscherschwankungen recht gut überein. Eine Untersuchung dieser Serie hat ergeben, daß die verzögerte Ausaperung zwischen 1886 und 1919 auf höhere Frühjahrsniederschläge, jene von 1954-1980 auf höhere Winterniederschläge zurückgeführt werden kann (PFISTER, 1985). Wenn wir, wie dies in den letzten zwei Jahrzehnten der Fall gewesen ist, eine Erwärmung der Winter mit einer Zunahme der Niederschläge gleichsetzen dürfen, dann läßt sich eine solche Entwicklung in der Untersuchungsperiode anhand der Daten von ALEXANDRE (1986) aufzeigen (vgl. Figur 2): im ganzen gesehen war der in der Periode 1525-1890 vorherrschende Typ des kalt-trockenen Winters auch für das Mittelalter charakteristisch. Eine erste Periode mit milderen (und feuchteren?) Wintern zeichnet sich um 1280, eine zweite zwischen 1330 und 1370 ab. Ein - allerdings bescheidenes - Vorrücken der Gletscherzungen in der ersten Hälfte des 14. Jahrhunderts erscheint von da her nicht ausgeschlossen. Der Aletschgletscher wäre dann um 1350 von einer etwas vorgeschobenen Ausgangslage aus vorgerückt. Wenn wir der Periode 13501375/80 den für die Jahre 1588-1610 nachgewiesenen Vorstoßbetrag von $70 \mathrm{~m} / \mathrm{Jahr}$ zugrunde legen, entspricht dies auch dem Ausmaß der von HOLZHAUSER (1984b) für das 14. Jahrhundert postulierten Zungenlängenänderung.

Zum Schluß eine Bemerkung zum möglichen humangeschichtlichen Stellenwert der vorliegenden Ergebnisse. Die Untersuchung der Periode 1525-1860 hat ergeben, daß die für die weitreichenden Gletschervor- 
Figur 2 Die Winter in Europa ( $\mathrm{N}$ der Alpen von 1020-1400) - fünfzigjährig gleitende Mittel der Häufigkeit milder und kalter Wintermonate

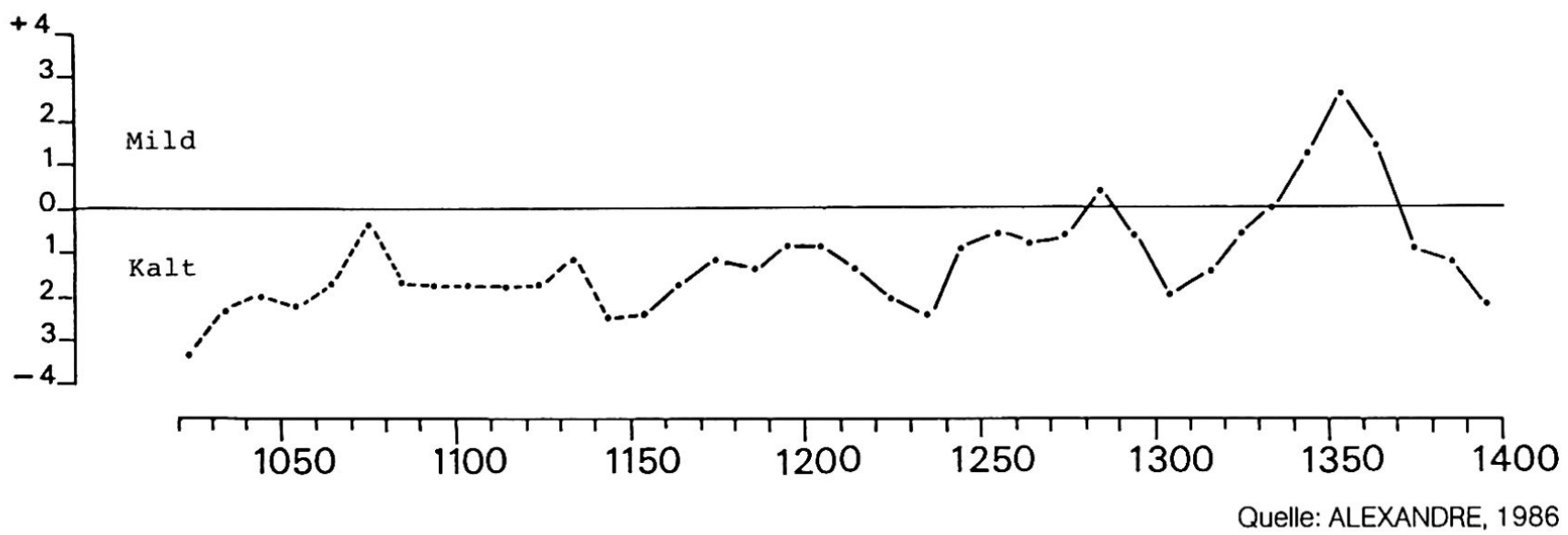

stöße maßgebenden klimatischen Impulse (1569-1573, 1586-1589, 1593-1597, 1627-1629, 1688-1694, $1769-1771,1812-1817,1851-1854)$ stets mit Subsistenzkrisen und einem mehr oder weniger großen Aderlaß an demographischer Substanz durch Ausfälle von Geburten und Übersterblichkeit verknüpft waren (PFISTER, 1984c: 60f). Dies scheint - mutatis mutandis - auch für das 14. Jahrhundert zu gelten: auf die Klimaschwankung von 1342-1347, die vielleicht härteste ökologische Belastungsprobe des letzten Jahrtausends, folgte der in vielen Teilen Europas schwerste demographische Aderlaß seit der Jahrtausendwende: die Pest von 1348-1350 (RuSSELL, 1978: 32). Bei der komplizierten Ätiologie dieser Krankheit, welche die Menschen noch heute verblüfft, kann aber die zeitliche Koinzidenz nicht als Beweis für Kausalität gelten. Zur Aufdeckung möglicher Zusammenhänge müßten gezielte Untersuchungen angesetzt werden.

\section{Zusammenfassung}

Die sommerlichen Witterungsverhältnisse der Periode 1270-1400 sind mit Hilfe von radio-densitometrischen Daten und quellenkritisch evaluierten anthropogenen Aufzeichnungen rekonstruiert und zum Vorstoß des Aletschgletschers im 14. Jahrhundert in Beziehung gesetzt worden. Das Zusammenspiel der beiden Datentypen ist zunächst in der gut dokumentierten Periode 1525-1860 überprüft worden. Anschließend sind die Temperatur- und Niederschlagsverhältnisse aufgrund übereinstimmender Angaben von radiodensitometrischen und anthropogenen Daten mit ungewichteten Indices beurteilt worden. Das Ergebnis stellt die Periode 1342-1347 als eine der nassesten und kältesten innerhalb des letzten Jahrtausends heraus. Es wird vermutet, daß diese Klimaschwankung für den in der Periode 1350-1380 vermuteten weitreichenden Vorstoß des Aletschgletschers den nötigen Schub geliefert hat und möglicherweise mit der europäischen Pestepidemie von 1348-50 verknüpft ist.

\section{Literatur}

ALEXANDRE, P. (1976): Le climat au Moyen Age en Belgique et dans les régions voisines (Rhénanie, nord de la France), Liège.

ALEXANDRE, P. (1986): Le climat en Europe au Moyen Age. Contribution à l'étude des variations climatiques de 1000 à 1425. Paris (Publ. de l'école des hautes études sociales).

AMBERG, B. (1890, 1892, 1897): Beiträge zur Chronik der Witterung und verwandter Naturerscheinungen mit besonderer Berücksichtigung auf das Gebiet der Reuß, der angrenzenden Gebiete der Aare und des Rheins. Jahresber. Höh. Lehranst., Luzern.

ARAGO, F. (1858): Sur l'état thermométrique du globe terrestre. Arago F., CEuvres complètes, Bd. 8, Paris, S. 184-646.

BELL, W., OGILVIE, A.E. (1978): Weather compilations as a source of data for the reconstruction of european climate during the medieval period. In: Climatic Change 1/4, S. 331-348.

BIDER, M., SCHUEPP, M., V. RUDLOFF H. (1958): Die Reduktion der 200jährigen Basler Temperaturreihe. Archiv für Meteorologie, Geophysik und Bioklimatologie, Serie B, 9/1, S. 360-412.

DARNAJOUX, H. (1976): Caractéristiques climatiques des saisons froides en France jusqu'à la fin du $X^{e}$ siècle. Secrétariat d'Etat aux Transports. Direction de la Metéorologie, Bibliographie signalétique hebdomadaire sélectionnée, Suppl. N. 8 , Paris.

DOUGLAS, K.S., LAMB, H.H. (1979): Weather observations and a tentative meteorological analysis of the period May to July 1958. Climatic Research Unit, Research Paper 6A.

EASTON C. (1928): Les hivers dans l'Europe occidentale. Leyden (Brill). 
FAIRBRIDGE, R.W. (1967): Climatic variations (Historical record). In: The Encyclopedia of Atmospheric Sciences and Astrology, New York, S. 205-211.

FLOHN, H. (1985): A critical assessment of proxy data for climatic reconstruction. In: The Climatic Scene, ed. by M.J. Tooley und G. M. Sheail, London (Allen and Unwin), S. 93-103.

Furrer G., et al. (1980): Zur Geschichte unserer Gletscher in der Nacheiszeit. Methoden und Ergebnisse. In: Das Klima. Analysen und Modelle. Geschichte und Zukunft, hg. von H. Oeschger, B.Messerli und M.Svilar, Berlin (Springer), S. 91-107.

GAMPER M., SUTER J. (1982): Postglaziale Klimageschichte der Schweizer Alpen. In: Geographica Helvetica, 37.Jg., S. 105-114.

HENNIG, R. (1904): Katalog bemerkenswerter Witterungsereignisse von den ältesten Zeiten bis zum Jahr 1800. In: Abh. des Kgl. Preuss. Meteor. Instituts, Bd. 2, Nr. 4, Berlin.

HOLZHAUSER, H. (1984a): Zur Geschichte der Aletschgletscher und des Fieschergletschers, Physische Geographie, Bd. 13, Zürich (Geographisches Institut).

HOLZHAUSER, H. (1984b): Rekonstruktion von Gletscherschwankungen mit Hilfe fossiler Hölzer. In: Geographica Helvetica, 39. Jg., S. 3-15.

INGRAM, M., UNDERHILL D., FARMER G. (1981): The use of documentary sources for the study of past climates. In: Climate and History, Ed. T. Wigley, Cambridge, S. 180-213.

LAMB, H.H. (1977): Climate: Present, Past and Future. Bd. 2, Climatic History and the Future, London (Methuen).

LE ROY LADURIE, E., BAULANT, M. (1980): Grape Harvests from the fifteenth through the ninetheenth centuries. In: J. of Interdisc. History, 10, S. 839-849.

LOETHER, H., Mc TAVISH, D.G. (1974): Descriptive statistics for sociologists. An Introduction, Boston, London (Allyn and Bacon).
MESSERLI P. (1979): Beitrag zur statistischen Analyse klimatologischer Zeitreihen, Geographica Bernensia G10, Bern (Geograph. Institut).

PFISTER, Ch. (1984a): Klimageschichte der Schweiz 1525-1860. Das Klima der Schweiz und seine Bedeutung in der Geschichte von Bevölkerung und Landwirtschaft, Bd.1, Bern (Haupt).

PFISTER, Ch. (1984b): Witterungsdatei Climhist. Bd. 1. Schweiz 1525-1863. Erhältlich bei Meteotest, Hallerstraße 12, $\mathrm{CH}$ 3012 Bern.

PFISTER, Ch. (1984c): Bevölkerung, Klima und Agrarmodernisierung 1525-1860. Das Klima der Schweiz von 1525-1860 und seine Bedeutung in der Geschichte von Bevölkerung und Landwirtschaft, Bd. 2, Bern (Haupt).

PFISTER, Ch., (1985): Snow cover, snow-lines and glaciers in Central Europe since the 16th century. In: The Climatic Scene, Ed. by M. J. Tooley und G. M. Sheail, London (Allen and Unwin), S. 154-174.

RUSSELL, J. C. (1978): Die Bevölkerung Europas 500-1500. In Europäische Wirtschaftsgeschichte, hg. von C.M. Cipolla und K. Borchardt, Stuttgart (Fischer), S. 13-43.

SCHWEINGRUBER, F.H., SCHAER E. und BRAEKER O.U. (1978): X-Ray densitometric results for subalpine conifers and their relationship to climate. Dendrochronology in Europe, Ed. by J.Fletcher, British Archaeological Reports, Internat. Series 51: 89-100.

SCHWEINGRUBER, F.H., BRAEKER O.U. und SCHAER E. (1979): Dendroclimatic studies on conifers from Central Europe and Great Britain. In: Boreas 8, S. 427-452.

SCHWEINGRUBER, F.H. (1983): Eine jahrringanalytische Studie zum Nadelbaumsterben in der Schweiz, Ber. der Eidg. Versuchsanstalt $f$. das Forstl. Versuchswesen, Nr. 235.

ZUMBÜHL, H.J. (1980): Die Schwankungen der Grindelwaldgletscher in den historischen Bild- und Schriftquellen des 12. bis 19. Jahrhunderts, Basel (Birkhäuser). 